\title{
Network Pharmacology Study of The Mechanism of Anti-Pneumonia Action Mechanism of Gentiana Rhodantha Franch
}

\author{
Jun Luo
}

Guizhou University Of Traditional Chinese Medicine First Affiliated Hospital

\section{Xiusheng Tang}

Zunyi Hospital of Traditional Chinese Medicine

\section{Guotao Shu}

Zunyi Medical University

\section{Dongxin Tang}

Guizhou University of Traditional Chinese Medicine First Affiliated Hospital

Jia Yu

Guizhou University of Traditional Chinese Medicine First Affiliated Hospital

\section{Jiangping Bao}

Guizhou University of Traditional Chinese Medicine First Affiliated Hospital

\section{Yanru Guo}

Guizhou University of Traditional Chinese Medicine

\section{Botao Chang}

Guizhou University of Traditional Chinese Medicine

\section{Danni Yang}

Guizhou University of Traditional Chinese Medicine First Affiliated Hospital Linjun Zhao ( $\square$ linjunzhao1010@163.com )

Xintian Community Health Service Center in Wudang District, Guiyang City https://orcid.org/00000001-5738-1156

\section{Research}

Keywords: Serum Dragon bile, Pneumonia, Network Pharmacology, Mechanism

Posted Date: June 2nd, 2021

DOl: https://doi.org/10.21203/rs.3.rs-539959/v1

License: (c) (1) This work is licensed under a Creative Commons Attribution 4.0 International License. Read Full License 


\section{Abstract}

Background: Serum dragon bile is a Chinese medicine used to treat pneumonia, but its mechanism of action is not clear. Meanwhile, due to the development of microarray and RNA-sequencing technology, high-throughput sequencing analysis is being used increasingly, and it has been applied as an indispensable tool in many medical fields. Therefore, in this article, we want to employed the bioinformatics approach to explore the relevant pharmacological mechanism of dragon serum bile in the treatment of pneumonia through network pharmacology.

Methods: In this paper, the active chemical composition and action target of serum dragon bile are obtained through the pharmacology database (TCMSP) of Chinese medicine system and the literature, and the data set of the intersection of active ingredient and disease target is established, and the protein interoperability network of serum gallbladder action target and pneumonia action target is analyzed by using protein interaction network (PPI). Using the Biological Information Annotation Database (DAVID) for gene ontology (GO) functional richness analysis and based on kyoto Gene and Genomics Encyclopedia (KEGG) pathogenic rich analysis, to predict the mechanism of the role of seroclon bile against pneumonia.

Results: Through the network pharmacological prediction, it is shown that the main chemical components of serum dragon bile are quercetin, isoorientin, luteolin, Stigmasterol, vanillic acid, etc, all of which have anti-pneumonia effects. The anti-pneumonia effect of serum dragon bile is mainly regulated by pathways in cancer, Bradder cancer, TNF signaling pathway, Hepatitis B and Non-small cell lung cancer, among which the TNF signaling pathway is more associated with pneumonia.

Conclusions: It is concluded from the network pharmacological prediction that serum dragon bile may play an anti-pneumonia role by promoting apoptosis, survival, immunity, etc. Its anti-pneumonia path is closely related to key targets IL6, FOS, CASP3 and AKT1. This study provides theoretical support for the follow-up study of the anti-pneumonia mechanism of serum gentian bile.

\section{Background}

Serum dragon bile (Gentiana rhodantha Franch.) from the dry whole grass of dragon bile plants, alias red dragon bile, small herring bile, star flower, dragon bile ${ }^{[1]}$,With the effect of dehumidification, detoxifying laxative fire and coughing, it is often used to treat wet hot jaundice, pulmonary fever cough and other diseases $^{[2]}$, and has added Chinese herbal varieties to the 2015 edition of the Chinese Pharmacopoeia. The addition of serum dragon bile to the Pharmacopeia indicates that its efficacy is fully recognized and has great potential in the development and application of monocetalycical and Chinese medicine. Staphylactic dragon bile is mainly distributed in southwest China, long-term as a local drug and ethnic drug use, has developed a variety of staphylactic dragon bile-related compound dosage forms, such as lung cough capsules, lung cough compounding agents, Kang women's capsules, etc ${ }^{[3-4]}$. Modern pharmacological studies have found that serum dragon bile has antibacterial, anti-inflammatory, 
antioxidant and other pharmacological activities ${ }^{[5]}$. Yao Haijoan ${ }^{[6]}$ compares different parts of the herbs, it was found that the antioxidant activity of the leaves, flowers and above ground parts was more prominent, and Liu Weiwen and others ${ }^{[7]}$ extracted positive 31 carbonane, positive 32 carbonane and positive 32 carbonic acid from the oil ether site of serum dragon bile, separate anti-TB bacteria experiments found that the petroleum ether extraction site of serum dragon bile has significant anti-TB bacteria efficacy, Xu et al ${ }^{[8]}$. Acetylcholinesterase inhibits activity, Wang Feiqing ${ }^{[9]}$ and other studies have found that serum dragon bile has a better therapeutic effect on mice infected with Streptococcus pneumoniae by lowering CRP, PCT, IL- 6 and IL-8 levels. Pulmonary cough compound composed of serum

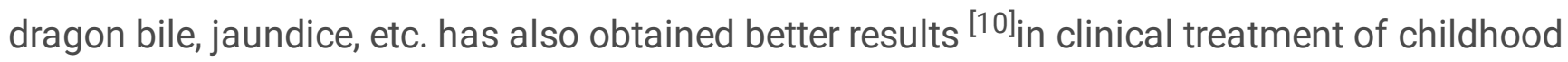
pneumonia. At present, there is no systematic study of its modern pharmacological activity.

Pneumonia refers to the inflammation of the airways, alba bubbles and pulmonary between the end of the disease, which can be caused by pathogenic microorganisms, immune damage, allergies and drugs, so its pathogenesis is more complex ${ }^{[11]}$. Traditional Chinese medicine in the treatment of pneumonia has a variety of factors, links, adjustment methods, can play a role in the occurrence of pneumonia, development of many links, but because of the multi-component, multi-target characteristics of the role of traditional Chinese medicine, it is difficult to clarify the basis and mechanism of the drug-effective substances. The rise of network pharmacology can provide a new method for the study of disease prevention and action mechanism of traditional Chinese medicine, which is different from the single research concept of "single drug corresponds to single target" in traditional pharmacology research, and can construct multi-level network by retrieving relevant databases, combining high-volume histological data analysis and computer simulation, and based on the "disease-gene-target-drug" interaction network, it can predict drug targets as a whole and systematically study the laws and mechanisms of drug interaction. Therefore, with the help of network pharmacology, this paper excavates the main active ingredients and targets of serum dragon bile anti-pneumonia, and provides a theoretical basis for the indepth study of the anti-pneumonia effect of serum dragon bile in the later period.

\section{Materials And Methods}

1.1 Acquisition of the active ingredient of serum dragon bile.

Finding the latest reported compounds of serum dragon bile by consulting the Serum Dragon Bile TCMSP database (http://tcmspw.com/tcmsp.php), and screen for active chemicals of sorptic dragon bile by screening for oral bio-utilization (OB) of $20 \%$ and drug-like (DL) of 0.15 , or with a documented active compound, screening out the active chemical composition of Gentiana gondii

1.2 Prediction of the target of the action of serum dragon bile and the target of pneumonia.

Using the component target prediction function in TCMSP, BATMAN and other databases, the effective components of serum dragon bile were collected. In the human gene database Genecards, human online Mendel genetic platform OMIM with "Pneumonia" as the key word, to retrieve the relevant target gene with 
pneumonia, and the active ingredient target gene mapping to screen out common targets, is the target of the treatment of pneumonia with the active ingredient of seroclon bile.

\subsection{Key target PPI network construction.}

By searching the string database online, 158 common targets were imported, the species was defined as "human" and a PPI network for the treatment of pneumonia was constructed. Export the results in TSV format, and then obtain the topological parameters of each target in the PPI network, Degree, Betweenness Centrality, Closeness Centrality, through the Cytoscape 3.6.1 software Network Analytic plug-in, calculate the median of the three topological parameters, and filter the above three topological parameter values to meet the targets that are greater than all medians or more than 2 times the median values respectively, listed as a table and visualizing the target ${ }^{[12]}$.

$1.4 \mathrm{GO}$ feature rich analysis and KEGG path rich analysis.

The filtered target points will be analyzed using the DAVID (https://david.ncifcrf.gov/) database for KEGG path analysis and GO (Gene Ontology) biological process analysis.

\subsection{Labeling of the KEGG signal path.}

Using the KEGG Mapper function in the KEGG (https://www.genome.jp/kegg/) signal pathway database, the target is marked on the signal pathway closest to pneumonia, verifying that serum gallbladce plays a multi-target, multi-pathway anti-pneumonia role.

\section{Results}

\subsection{Screening of active ingredients.}

By consulting the relevant literature, 79 compounds were obtained, 79 compounds were screened, the ADME parameters (OB-20\% and DL-0.15) were used as the criteria, or the activity of the compound was reported in the existing literature, a total of 6 active compounds were screened, the results are seen in Table 1.

Table 1 Number of serum gallblader active compounds and targets. 


\begin{tabular}{|c|c|c|c|c|}
\hline Molld & MolName & $\mathrm{OB} \otimes \% \bigotimes$ & $\mathrm{DL}$ & Target numbers \\
\hline MOL000098 & quercetin & 46.43 & 0.28 & 135 \\
\hline MOL000498 & isoorientin & 23.30 & 0.76 & 26 \\
\hline MOL000006 & luteolin & 36.16 & 0.25 & 53 \\
\hline MOL000449 & Stigmasterol ${ }^{[13]}$ & 43.83 & 0.76 & 24 \\
\hline MOL000114 & vanillic acid $^{[14]}$ & 35.47 & 0.04 & 8 \\
\hline MOL000511 & ursolic acid & 16.77 & 0.75 & 80 \\
\hline
\end{tabular}

2.2 Predictive results of the potential target for the treatment of pneumonia by serum dragon bile.

In the Genecards and OMIM databases, using "Pneumonia" as the key word, a total of 3980 genes related to pneumonia were retrieved, the pneumonia gene was matched with the target associated with serum dragon bile, and the results were shown in Figure 1. A total of 158 common targets were obtained, indicating that the 158 targets (PTGS1, AR, PPARG, PTGS2, ADRB2, etc.) were involved in the common system of serum gallbladler active ingredient pneumonia, and these 158 targets were common targets.

\subsection{PPI network analysis.}

The network diagram of the 158 interaction relationship obtained in the String database was imported into the string database, the species was set to "human" and the confidence level was 0.950 , as shown in Figure 2. Export the results in TSV format and import the TSV files into the Cytoscape 3.6.1 software. Using the plug-in Network Analytics to get topological parameters Foregree, Betweenness Centrality, Closeness Centrality for each target, where number of nodes $=158$, number of edges $=272$, average node $=$ degree $=3.44$, PPI enrichment $p$-value $\triangle 1.0 \mathrm{e}-16$, calculate the median of the three topology parameters are $27,0.0021,0.5259$, filter Degree, Betweenness centrality are greater than 2 times the median, Closeness centrality is greater than the median target.

A total of 30 key targets were obtained from the screening results. The results are shown in Table 2. The interaction between proteins shows that these targets are interconnected and play an anti-pneumonia effect through multi-channel and multi-faceted coordination. By counting the number of occurrences of each target, that is, the number of gene connection nodes, the more the number of connection nodes, the more important the role of the target in the treatment of pneumonia, indicating that these targets are important in the PPI network The effect suggests that these targets have important significance in the effect of safflower gen.

Table 2. Related topological parameters of the targets of the anti-pneumonia active ingredients of Gentiana 


\begin{tabular}{|c|c|c|c|c|c|}
\hline \multirow[t]{2}{*}{ UniprotID } & \multirow[t]{2}{*}{ Protein names } & \multirow[t]{2}{*}{ name } & \multirow[t]{2}{*}{ Degree } & \multirow{2}{*}{$\begin{array}{l}\text { Betweenness } \\
\text { Centrality }\end{array}$} & \multirow{2}{*}{$\begin{array}{l}\text { Closeness } \\
\text { Centrality }\end{array}$} \\
\hline & & & & & \\
\hline P31749 & $\begin{array}{l}\text { RAC-alpha serine/threonine- } \\
\text { protein kinase }\end{array}$ & AKT1 & 107 & 0.0598 & 0.7548 \\
\hline P02768 & Serum albumin & ALB & 101 & 0.0718 & 0.7371 \\
\hline P05231 & Interleukin-6 & IL6 & 99 & 0.0423 & 0.7202 \\
\hline P15692 & $\begin{array}{l}\text { Vascular endothelial growth factor } \\
\text { A }\end{array}$ & VEGFA & 94 & 0.0262 & 0.7040 \\
\hline P35354 & Prostaglandin G/H synthase 2 & PTGS2 & 88 & 0.0334 & 0.6856 \\
\hline P42574 & Caspase-3, CASP-3, & CASP3 & 87 & 0.0280 & 0.6886 \\
\hline P00533 & Epidermal growth factor receptor & EGFR & 85 & 0.0302 & 0.6767 \\
\hline P05412 & Transcription factor AP-1 & JUN & 83 & 0.0191 & 0.6709 \\
\hline P01133 & Pro-epidermal growth factor & EGF & 82 & 0.0265 & 0.6653 \\
\hline P28482 & Mitogen-activated protein kinase 1 & MAPK1 & 82 & 0.0292 & 0.6681 \\
\hline P01106 & Myc proto-oncogene protein & MYC & 81 & 0.0183 & 0.6653 \\
\hline P10145 & Interleukin-8, IL-8 & CXCL8 & 80 & 0.0162 & 0.6624 \\
\hline P14780 & $\begin{array}{l}\text { Matrix metalloproteinase-9, MMP- } \\
9\end{array}$ & MMP9 & 80 & 0.0186 & 0.6624 \\
\hline P01584 & Interleukin-1 beta, IL-1 beta & IL1B & 75 & 0.0114 & 0.6488 \\
\hline P03372 & Estrogen receptor, ER & ESR1 & 75 & 0.0186 & 0.6488 \\
\hline P24385 & G1/S-specific cyclin-D1 & CCND1 & 73 & 0.0155 & 0.6382 \\
\hline P01100 & Proto-oncogene c-Fos & FOS & 72 & 0.0198 & 0.6408 \\
\hline P13500 & C-C motif chemokine 2 & CCL2 & 71 & 0.0096 & 0.6356 \\
\hline P22301 & Interleukin-10, IL-10 & IL10 & 66 & 0.0069 & 0.6181 \\
\hline P08253 & 72 kDa type IV collagenase & MMP2 & 66 & 0.0109 & 0.6206 \\
\hline P04626 & $\begin{array}{l}\text { Receptor tyrosine-protein kinase } \\
\text { erbB-2 }\end{array}$ & ERBB2 & 63 & 0.0110 & 0.6157 \\
\hline Q04206 & Transcription factor p65 & RELA & 62 & 0.0130 & 0.6038 \\
\hline P37231 & $\begin{array}{l}\text { Peroxisome proliferator-activated } \\
\text { receptor gamma, PPAR-gamma }\end{array}$ & PPARG & 61 & 0.0105 & 0.6157 \\
\hline 015519 & $\begin{array}{l}\text { CASP8 and FADD-like apoptosis } \\
\text { regulator }\end{array}$ & CASP8 & 57 & 0.0087 & 0.6015 \\
\hline
\end{tabular}




\begin{tabular}{|llllll|}
\hline Q07817 & Bcl-2-like protein 1, Bcl2-L-1 & BCL2L1 & 56 & 0.0059 & 0.6015 \\
\hline P09601 & Heme oxygenase 1 & HMOX1 & 56 & 0.0099 & 0.6015 \\
\hline P05362 & $\begin{array}{l}\text { Intercellular adhesion molecule 1, } \\
\text { ICAM-1 }\end{array}$ & ICAM1 & 56 & 0.0028 & 0.5970 \\
\hline P05067 & $\begin{array}{l}\text { Amyloid-beta precursor protein, } \\
\text { APP }\end{array}$ & APP & 56 & 0.0320 & 0.5992 \\
\hline P05121 & $\begin{array}{l}\text { Plasminogen activator inhibitor 1, } \\
\text { PAl, PAl-1 }\end{array}$ & SERPINE1 & 55 & 0.0113 & 0.6015 \\
\hline P05112 & Interleukin-4, IL-4 & IL4 & 55 & 0.0073 & 0.5925 \\
\hline
\end{tabular}

\subsection{GO Biology Feature Rich Analysis}

A total of 158 common targets of the serum dragon bile compound target and pneumonia-related target were analyzed for $\mathrm{GO}$ richness, and the top 5 riching processes include response to drug, signal transduction, positive regulation of response to lipopolysaccharide, negative regulation of apoptotic process and inflammatory response. The rich results were all associated with the top 30 genes, such as IL-6, IL-10 and FOS, and the top 20 biological processes were presented in the form of bubble charts, as shown in Figure 3. Quick Go's interpretation of response to drug refers to any process that causes changes in the body's state or activity (movement, secretion, enzyme production, gene expression, etc.) caused by lipid polysaccharose stimulation. Inflammatory response refers to the immediate defensive response of vertebrate tissue to infection or injury caused by chemical or physical substances, characterized by local vascular dilation, plasma seepage into cell gaps, and white blood cells and macrophages, which are similar to inflammatory reactions. The Cytoscape 3.6.1 software plug-in "Bingo" was imported into the Serum Dragon Bile Composite Target, and the species was set as "human", and $P<1.0 \times 10^{-11}$ was taken for visual analysis of biological processes. The yellow circle represents the biological process of the adjusted $P<1.0 \times 10^{-11}$, and the size of the circle indicates how much of the relevant target is well-connected in the path, as shown in Figure 4. The darker the color of the circle represents the stronger the concentration effect, reflecting that the mechanism of action of seroclon bile against pneumonia involves multiple biological processes in the body, and the various biological processes are related to each other, and also indicates that the active ingredient of serococcal bile may play an anti-pneumonia role by regulating these biological processes.

\subsection{KEGG path analysis}

The 158 potential targets were mapped to the database for KEGG path agglus rich analysis, and a total of 113 signal paths were obtained. By screening KEGG rich analysis $P \leq 0.000001, \mathrm{FRD}<0.001$ screened out a total of 29 signal pathways of major richness of key targets, which are closely related to the mechanism of the role of red dragon bile against pneumonia, including Pathways In cancer, Bradder cancer, Hepatitis B, TNF signaling pathway, Prostate cancer and Non-small cell lung cancer, etc., are 
associated with treatments such as bladder cancer, liver cancer, prostate cancer, etc., and the first 20 pathways with significant differences are output in the form of bar charts, as shown in Figure 5.

Using Cytoscape 3.6.1 software, 158 common genes, diseases and 6 active ingredients were visualized and analyzed to create an interactive network of serum gallblades against pneumonia. After screening out the corresponding interactive proteins and visualizing them with different color and shape graphs, the network relationship between the active chemical composition and the target can be seen intuitively. The results can be seen in Figure 6 .

\section{Discussion}

Sedgy dragon bile in antibacterial, treatment of pneumonia and other aspects have a better therapeutic effect, but at present, the mechanism of serum dragon bile anti-pneumonia is not clear. Through the network pharmacological prediction, it is shown that the main chemical components of serum dragon bile are quercetin, isoorientin, luteolin, Stigmasterol, vanillic acid, etc, all of which have anti-pneumonia effects. Quercetin, for example, inhibits MAPK signaling path expression by regulating genes such as p38, thereby reducing the effect of intervention ${ }^{[15]}$. These active anti-pneumonia ingredients are mainly related to genes such as IL6, FOS, CASP3, AKT1 on the TNF signaling pathway pathway.

In this study, the IL-6 gene in the key target screening Degree, Closeness Centrality and Betweenness Centrality are larger, the overall ranking is higher, with a strong correlation with pneumonia, is one of the key targets of sorobacterial bile anti-pneumonia. IL-6 is a multi-effect cytokine, according to the study of inflammation since IL- 6 is often used as an indicator of inflammation, IL-6 level increase is the earliest infectious indicators. Mainly produced by monocyte macrophages, endodertic cells and lymphocytes, it is a multi-functional cytokine ${ }^{[16]}$ that has been studied more in recent years. Currently, there are studies on the complex inflammatory cell IL-6 that constitutes COPD airway inflammation, and IL- 6 can promote and accelerate the development of local inflammation, which eventually leads to the remodeling of airway structure and the formation of airway obstruction ${ }^{[17]}$. Through KEGG map, it can be found that TNF signaling pathway is mainly composed of signal pathways such as MAPK signaling pathway, NF-kappa B signaling pathway and PI3-Akt signaling pathway, which can induce apoptosis, survival, Inflammation and immunity, an important factor in intracellular signaling pathways, TNF, is activated and binds to its subjects TNFR1, TNFR2, resulting in terabytes of TNFR1 or TNFR2. TNFR1 is the main subject of TNF, also known as TNF-a, expressed by almost all cells. In limited cells, TNFR2 is expressed as a subject of TNF and LTA, which binds to proteins such as TRADD or TRF2 under the guidance of TNFR after ligation, resulting in the collection of signal transdutors ${ }^{[18]}$. TNFR1 signal transducting induces the activation of a variety of genes, mainly controlled by two different pathways, NF-kappa B and MAPK cascading, namely apoptosis and necrosis. The TNFR2 signal activates the NF-kappa B path, including the PI3K-dependent NF-kappab path and the JNK path ${ }^{[19]}$. Such as external stimulation signals stimulate activation of the IKK protein complex, IKK complex phosphate activation of I-kB and other proteins, I-kB by phosphate and then ubially, and finally binding to the $\mathrm{kB}$ part of a specific target gene, starting the transcription of the target 
gene ${ }^{[20]}$. PI3-Akt signaling pathway promotes IkBa by regulating IKKs key genes, IKKs key genes phosphate, blocks the expression of NF-kB, and also promotes the occurrence of cell survival, specifically regulating the pathway as shown in Figure 7. Therefore, serum dragon bile may play an anti-pneumonia role by promoting apoptosis, survival, immunity, etc. This paper provides the relevant basis for the study of the mechanism of the anti-pneumonia action of serum dragon bile in the later period, but the specific mechanism of serum gallbladce against different pneumonia needs further study and verification.

\section{Conclusions}

It is concluded from the network pharmacological prediction that serum dragon bile may play an antipneumonia role by promoting apoptosis, survival, immunity, etc. Its anti-pneumonia path is closely related to key targets IL6, FOS, CASP3 and AKT1. This study provides theoretical support for the follow-up study of the anti-pneumonia mechanism of serum gentian bile.

\section{Declarations}

\subsection{Ethics approval and consent to participate}

Not applicable

\subsection{Consent for publication}

Not applicable

\subsection{Availability of data and materials}

All data generated or analysed during this study are included in this published article and its supplementary information files.

\subsection{Competing interests}

The authors declare that they have no competing interests .

\subsection{Funding}

National Administration of Traditional Chinese Medicine Speciality Technology Heritage Talent Project (National Chinese Medicine Office Letter No. 2018, No. 204), Guizhou Provincial Science and Technology Department Guizhou Province high-level innovative talent training program (100 levels) 4032, Guizhou Provincial Organization Department of Guizhou Province, Chinese medicine tumor inheritance and scientific and technological innovation talent base (Yu people lead the hair of the No. 3), Guizhou Province Science and Technology Program Project (Yuke Joint Support , 2018, 2772), Guizhou Provincial Science and Technology Department guizhou Chinese medicine tumor inheritance and scientific and technological innovation talent team (Yuke joint platform talent , 2020 , 5013), Guiyang City Science and 
Technology Bureau, Guizhou University of Traditional Chinese Medicine, the first affiliated hospital big health science and technology cooperation project (Tsuko contract 2019, 9-2-11), Guizhou Provincial Administration of Traditional Chinese Medicine, Chinese medicine prevention and control of new crown pneumonia special research project (QZYYXG-2020-1). The central government guides local science and technology development funding projects(No.4001区2020区.

\subsection{Authors' contributions}

Jun Luo and Xiusheng Tang have equally contributed to the manuscript to concept the study design, literature search, data analysis, data interpretation, and article drafting. Guotao Shu, Jia Yu and Yanru Guo contributed to data interpretation and manuscript critical revisions. Dongxin Tang, Botao Chang and Linjun Zhao contributed to concept the study design, data analysis, data interpretation, and manuscript critical revision. All authors read and approved the final version of the manuscript.

\subsection{Acknowledgements}

Not applicable in this section.

\subsection{Author details}

Luo Jun, director pharmacist, engaged in Chinese medicine, ethnic medicine quality control and new drug research, E-mail: 53011402@qq.com.

Zhao Linjun, in charge of pharmacists, engaged in chinese medicine, ethnic medicine quality control and new drug research, E-mail:93343673@qq.com, linjunzhao1010@163.com.

\section{References}

1. The Editorial Board of Chinese Botany of the Chinese Academy of Sciences. Chinese Botany: Volume 62: Beijing: Science Press, 1988: 148-150.

2. National Pharmacopeia Commission. Pharmacopoeia of the People's Republic of China: One. Beijing: China Medical Science and Technology Press, 2015:151.

3. Revolutionary Committee of Yunnan Provincial Health Bureau. Yunnan Chinese Herbal Medicine ... Kunming: Yunnan People's Press, 1971:570-571.

4. New anti-cough phlegm medicine: pulmonary cough enseller. Central and Southern Pharmacology, 2009, (7): 554-556.

5. Fang Yumei, Zhang Jiaman, Sun Aiqun, etc. Miao medicine syringe dragon bile in-body antibacterial test. Northern Gardening, 2018, (7) : 28-132.

6. Yao Haijun. Study on the chemical composition of serum dragon bile. Shanghai: Shanghai University of Traditional Chinese Medicine, 2015. 
7. Liu Weiwen, Liu Ping, Wang Ruizhen, et al. Research on the active components of dragon bile gallbladder against tuberculosis. Chinese Journal of Chinese Medicine, 1985, (12): 35-36.

8. Xu M, Zhang M, Wang D, et al. Phenolic compounds from the whole plants of Gentiana rhodantha (Gentianace)[J]. Chemistry and Biodiversity, 2011, 8:1891.

9. Wang Feiqing, Tao Yuxi, Li Society, etc. Streptococcus gallblades on the prevention and treatment of streptococcus pneumoniae pneumoniae. China Public Health, 2018, 11:1484-1486.

10. Marina, Qin Lihui. Observation of the clinical effects of pulmonary cough adhesive in the treatment of acute bronchitis in children. Straits Pharmacy, 2019, 01:162-163.

11. Chen Willlei. Clinical care for patients with pneumonia. China Disability Medicine, 2013, 21 (4): 309.

12. Xu Feng, Huang Xulong, Zhang Mei, et al. Study on the mechanism of anti-inflammatory action of large blood vine based on network pharmacology. Journal of Chinese Medicine, 1-12.

13. Aaron Opoku Antwi, David Darko Obiri, Newman Osafo. Stigmasterol Modulates Allergic Airway Inflammation in Guinea Pig Model of Ovalbumin-Induced Asthma(Stigmasterol[J]. Mediators Inflamm, 2017:2953930.

14. Bai F, Fang LY, Hu HZ.Vanillic Acid Mitigates the Ovalbumin (OVA)-induced Asthma in Rat Model Through Prevention of Airway Inflammation[J]. Biosci Biotechnol Biochem, 2019, 83(3):531-537.

15. Cheng Jian, Jiang Yuhua. Quercetin reduces radioactive pneumonia in mice by inhibiting the expression of the active protein kinase signaling path of fissurer. Journal of Shandong University (Medical Edition), 2019, 5:87-92.

16. Pan Linghui. Advances in the study of the role of cytokine balance in inflammatory responses. Medical Review, 2005, 9:775-777.

17. Zhang Wei. The interventional effect and mechanism of a gum and jaundol on COPD model mice and pulmonary fibrosis model mice were studied. Shandong University of Traditional Chinese Medicine, 2018.

18. Qi Zhilin. Study on molecular mechanisms of phosphate HSP27 against TNF-a induced apoptosis of HeLa.

19. High purity. HSPA13 Regulates NF-B Activation and Programd Death Response induced by TNF alpha . Zhejiang University, 2018.

20. Meng Jieru. Effects of TNFR expression and nrhTNF on tumor cell growth and NF-B activity in tumor cell.Fourth Military Medical University, 2002.

\section{Figures}




\section{Gentiana rhodant}

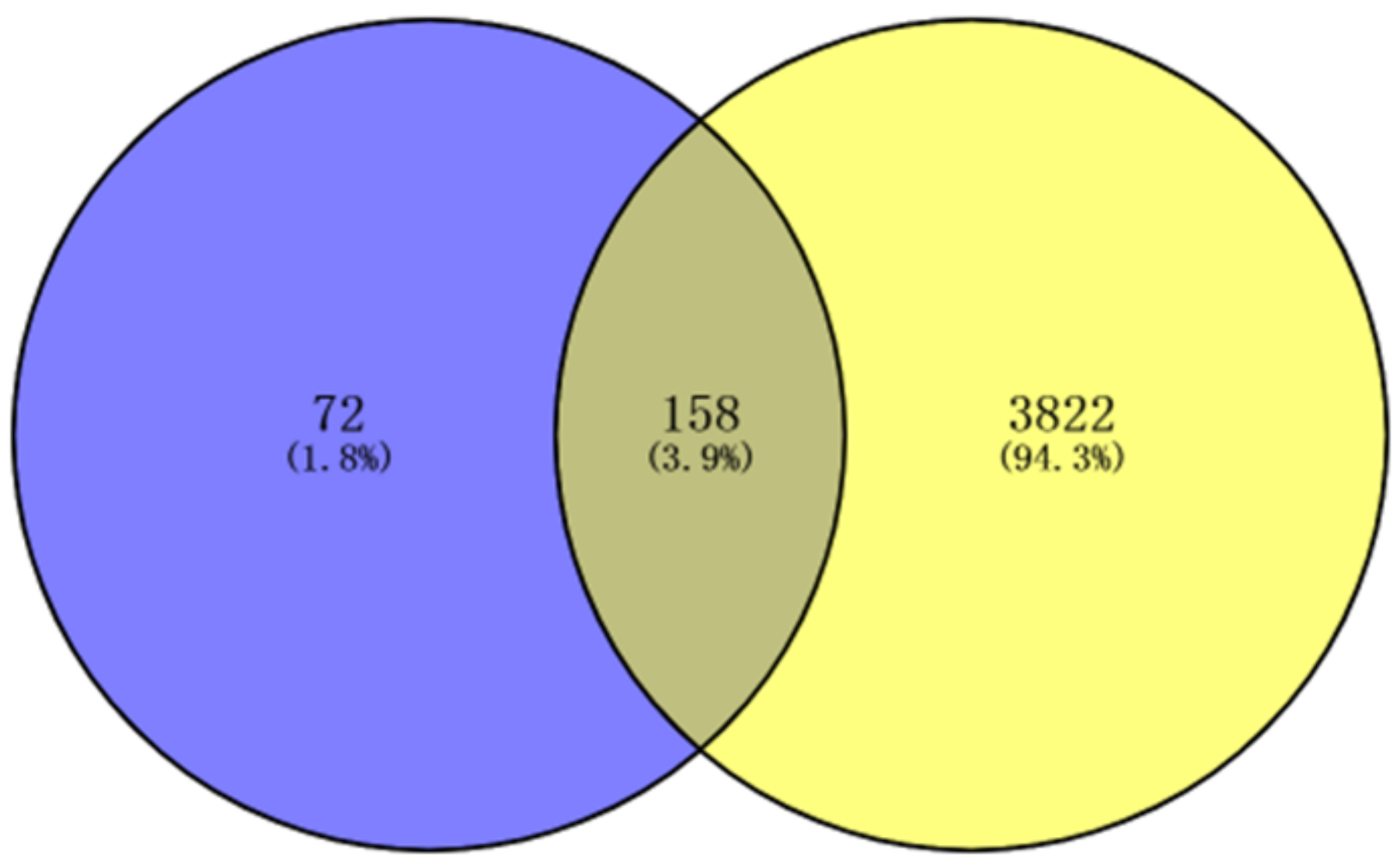

Figure 1

Serum Dragon Bile - Pneumonia Intersection Target Map. Pneumonia genes and gentian related targets are intersected, and there are 158 common targets in total. 


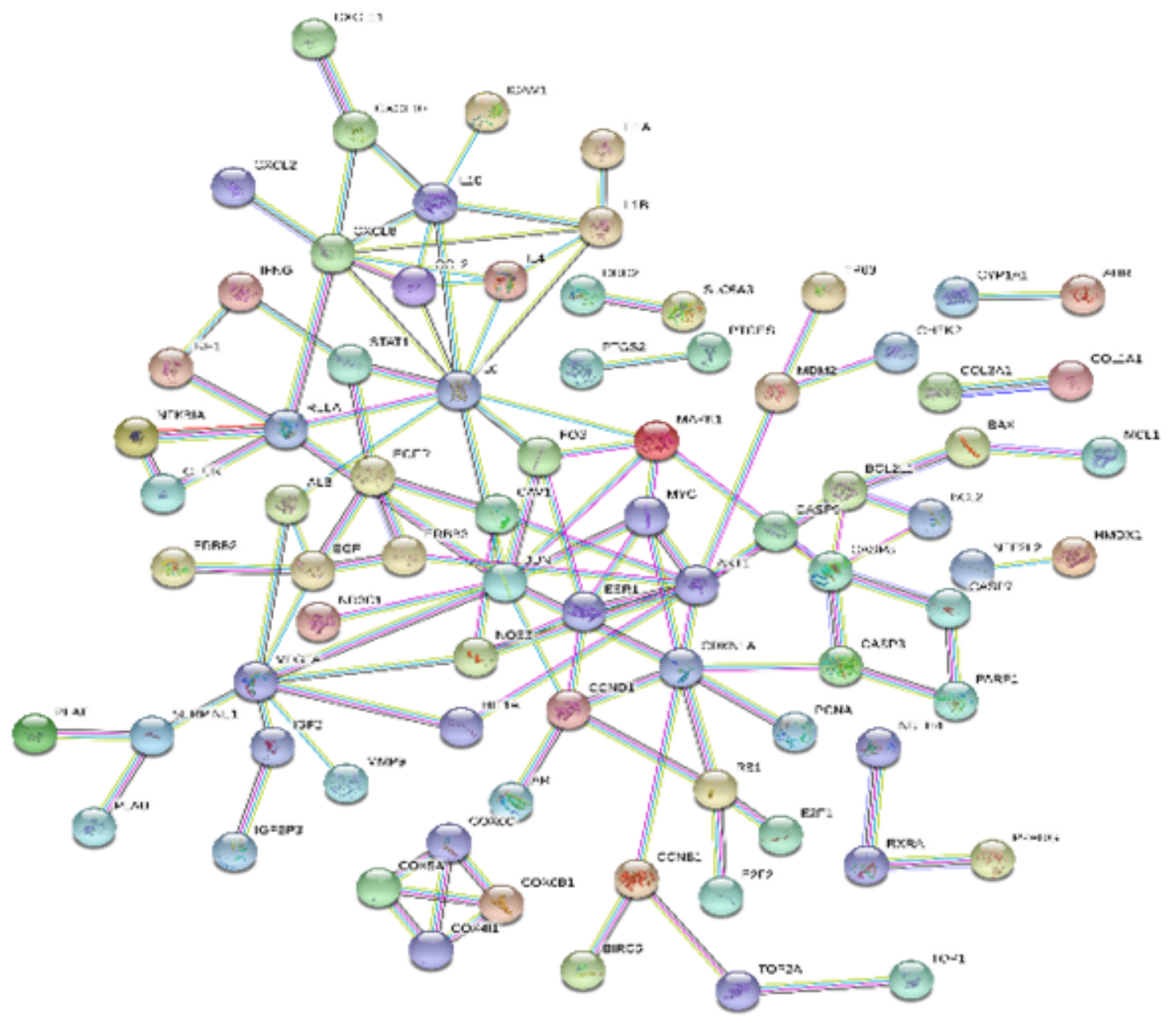

Figure 2

PPI network diagram of the anti-pneumonia target of Gentiana saffron. After importing 158 common target genes into the String database, the related protein-protein interaction network diagram is obtained 


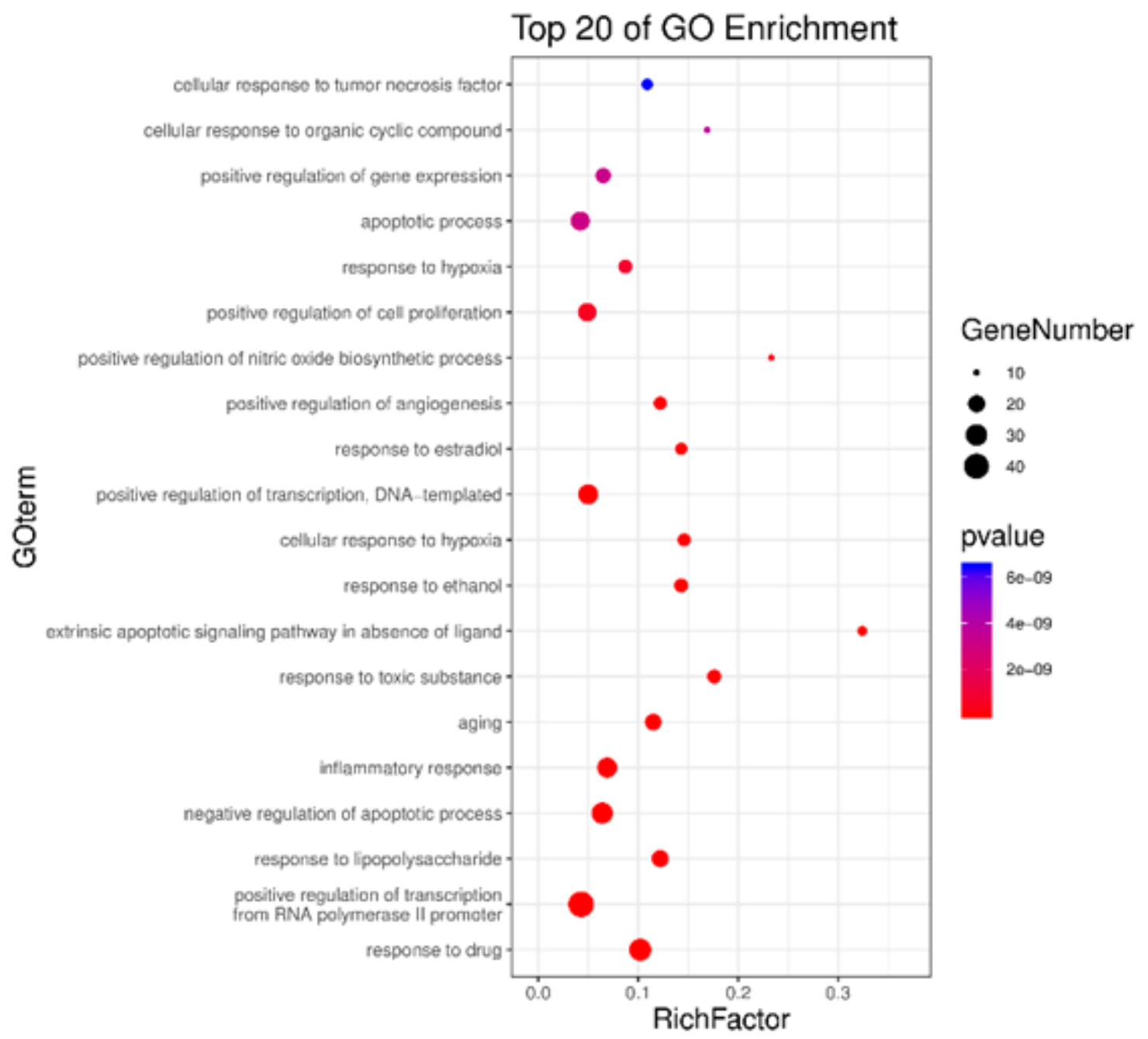

Figure 3

GO Biology Feature-Rich Analysis Bubble Map. Perform GO analysis on 158 common target genes, select the top 20 for mapping. 


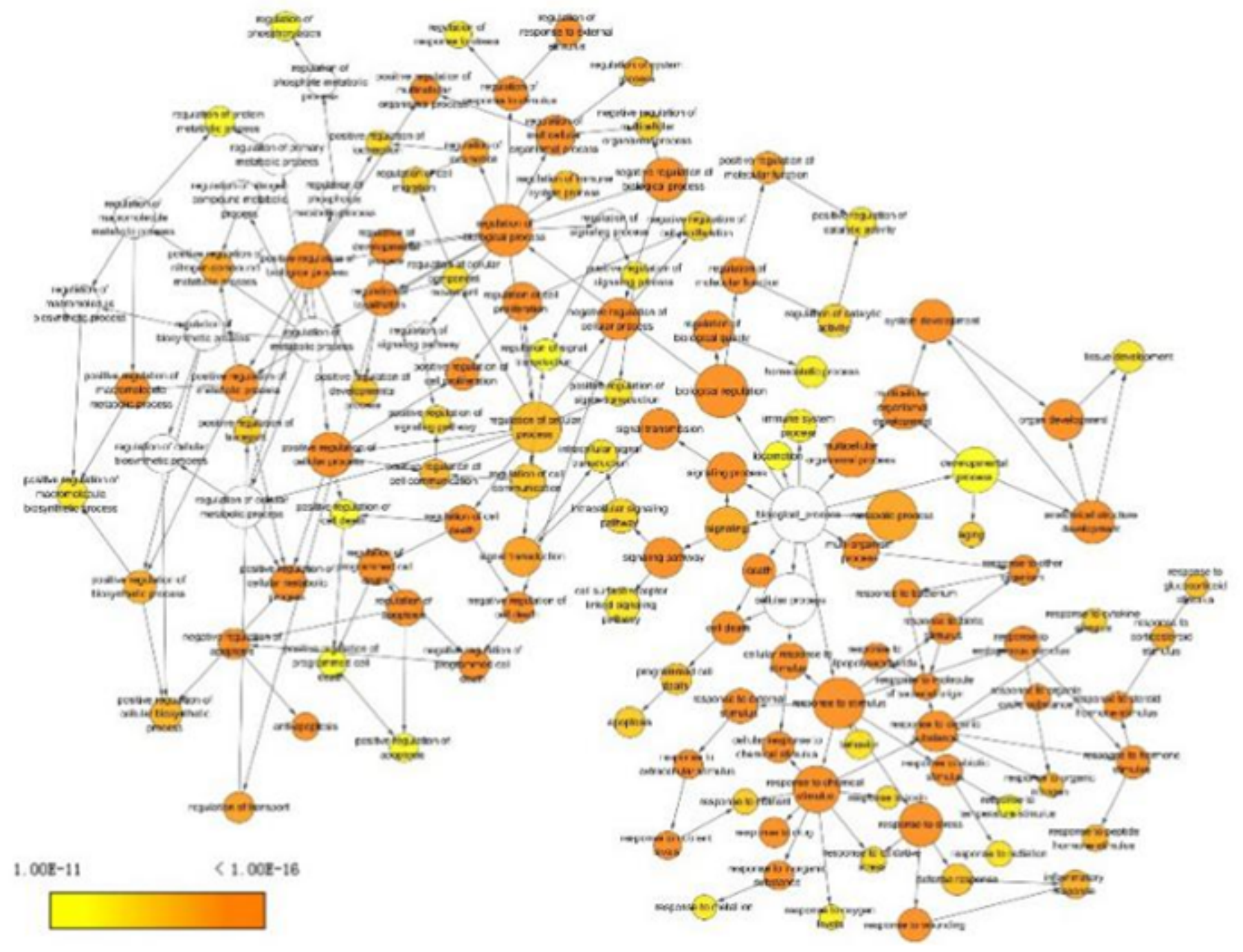

Figure 4

Map of the biological processes of serum dragon bile-pneumonia. Import common target genes to the Cytoscape 3.6.1 software, then set as "human", and $\mathrm{P}<1.0 \times 10-11$ was taken for visual analysis of biological processes. The yellow circle represents the biological process of the adjusted $\mathrm{P}<1.0 \times 10-11$, and the size of the circle indicates how much of the relevant target is well-connected in the path. 


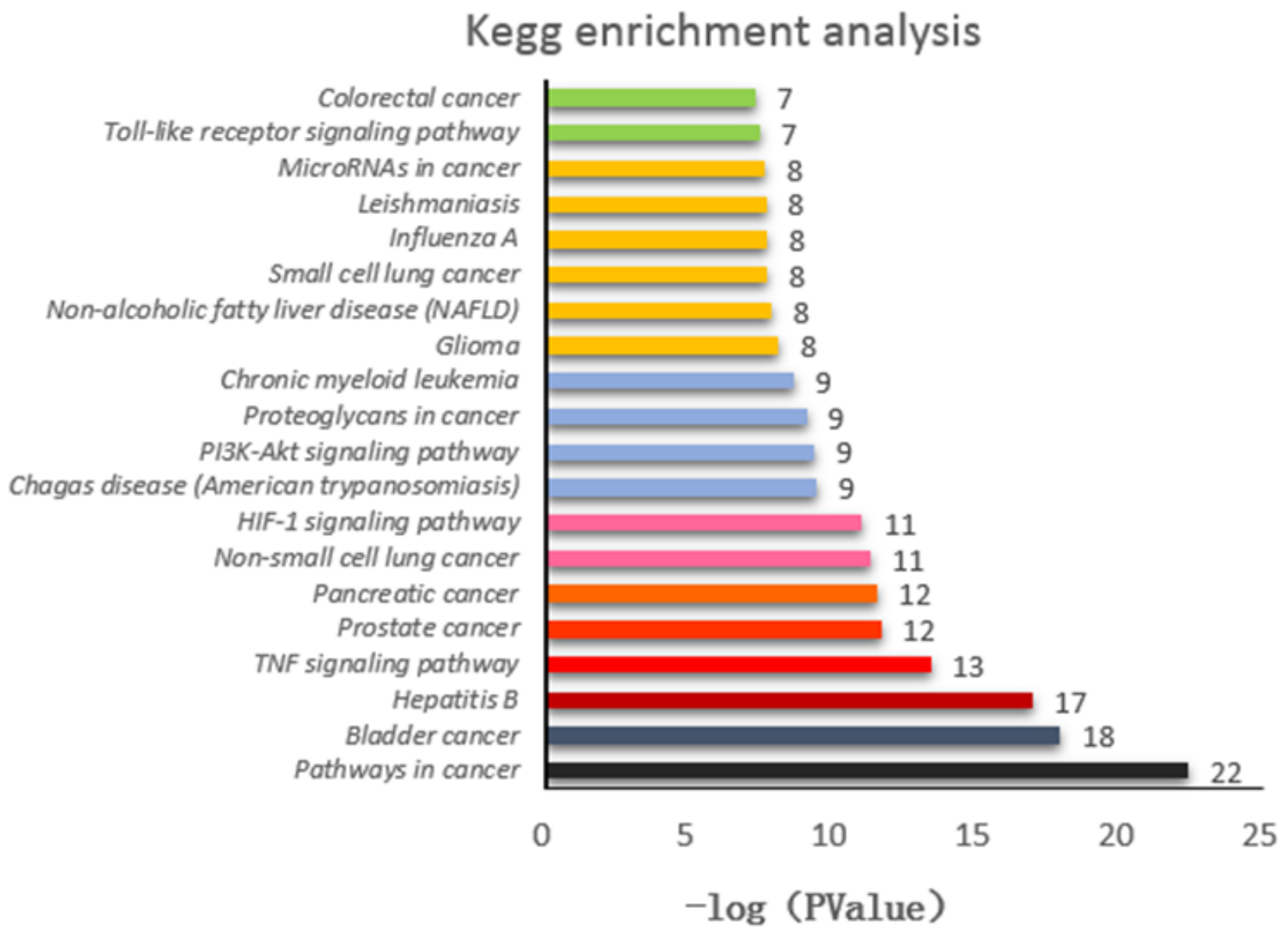

Figure 5

KEGG Rich Analysis Bar Chart. The KEGG pathway enrichment analysis was performed on 158 potential targets, and a total of 113 signal pathways were obtained. These are closely related to the antipneumonia mechanism of serum dragon bile. 


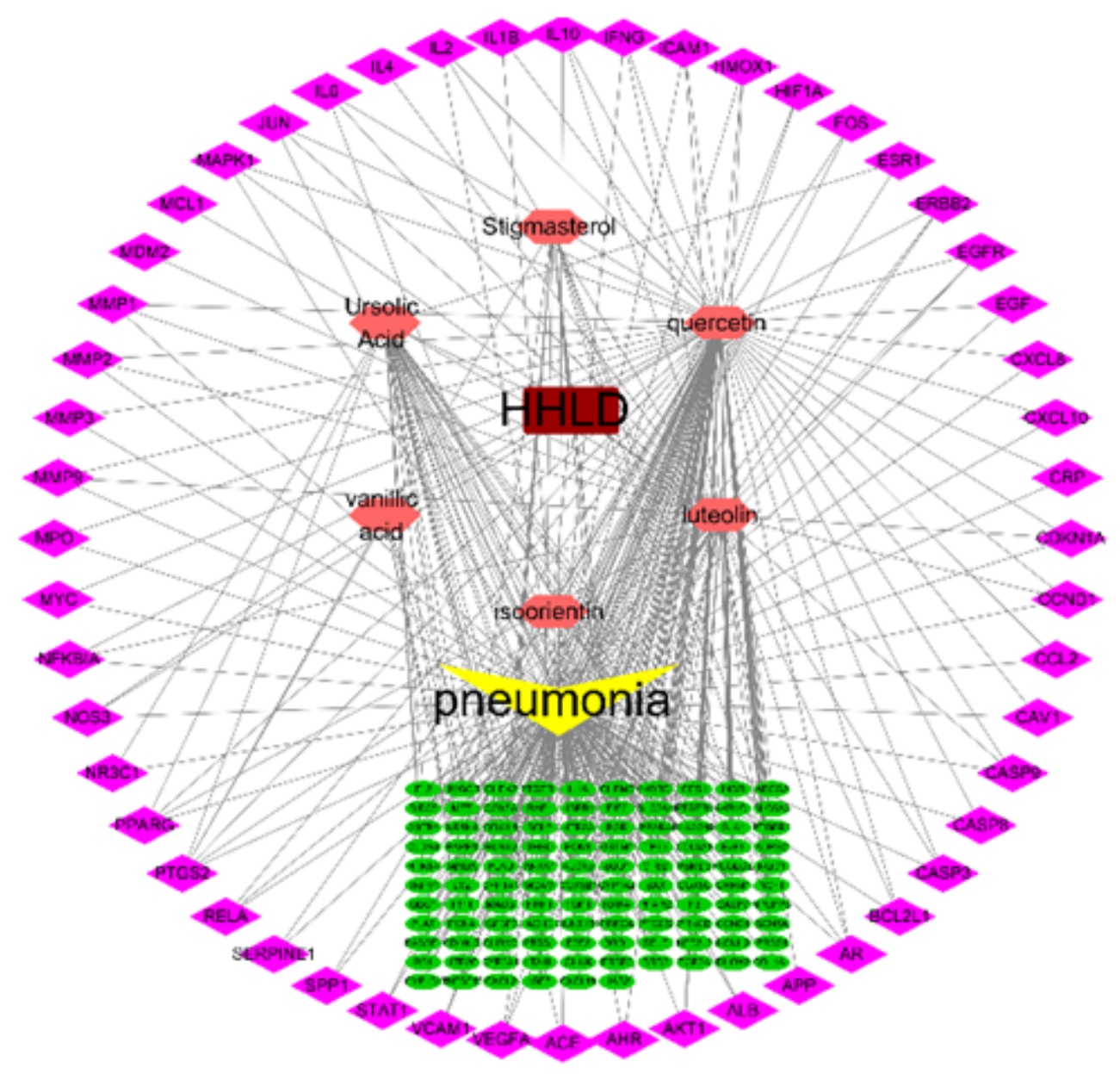

Figure 6

Serum dragon bile-target-gene-disease network map. There are 52 pink prism genes, representing the top 50 genes out of 158 genes, green has 106, representing the longer ranked genes, yellow for disease, crimson for drugs, the drug peripheral 6 chemical components, the results show that the greater the shape of the connection. 
TNF SIGNALING PATHWAY
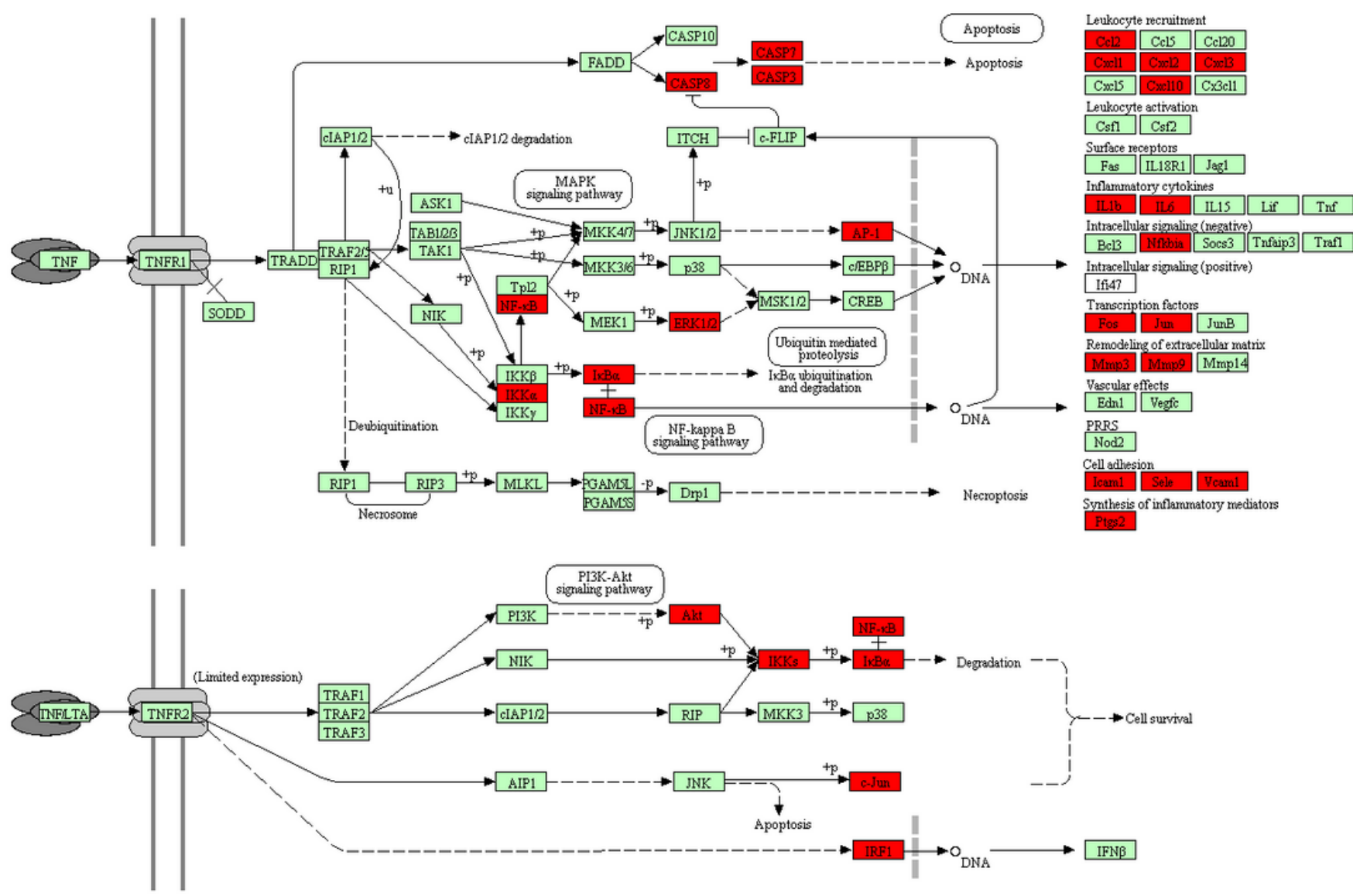

$0466877 / 5119$
(c) Kanehisa Laboratonies

\section{Figure 7}

TNF signaling pathway target annotation diagram. TNF interacts with a variety of genes to regulate the occurrence of inflammation. 